\title{
Vibrações Livres e a Função de Green Temporal para um Modelo de Euler-Bernoulli
}

M.K. GIARETA ${ }^{1}$, Departamento de Matemática, Universidade de Passo Fundo, 99.010-070 Passo Fundo, RS, Brasil

J.R. CLAEYSSEN², Instituto de Matemática - PPGMAp/Promec, Universidade Federal do Rio Grande do Sul, Cx.P. 10673, 90.001-970, Porto Alegre, RS, Brasil.

Resumo. Este trabalho visa a obtenção da resposta impulso, ou função de Green temporal para uma viga longa e fina descrita pela equação de Euler-Bernoulli sob a influência de uma força axial. Simulações para a função de Green temporal são apresentadas para vigas fixas-livre, engastadas, e deslizante-apoiada.

\section{O Modelo de Euler-Bernoulli}

Este trabalho visa a derivação de uma formulação sistemática para a resposta livre de um modelo elástico Euler-Bernoulli, que descreve a dinâmica de uma viga, em termos da base temporal, gerada pela resposta impulso ou, função de Green no tempo e a determinação desta última de maneira espectral. Os modos flexurais do sistema podem ser calculados com o uso da base clássica de Euler ou, com a introdução de uma base, chamada de base dinâmica espacial, a qual é melhor condicionada diante variação dos parâmteros físicos. Neste problema são consideradas condições de contorno genéricas. As simulações foram realizadas com auxílio do software Maple.

A viga em estudo está sujeita ao efeito de uma força paralela ao eixo horizontal, além da força lateral já existente. A Figura 1 mostra o esquema de uma viga fixalivre de comprimento $L$, onde $v(t, x)$ é o deslocamento transversal modificado por uma força axial $N$ e $f(t, x)$ é a força externa ou carga. É assumido por simplicidade que a força axial é constante e que o equilíbrio transversal não é afetado pela força axial. Segundo a teoría de de Euler Bernoulli, a qual supõe que o cisalhamento e a inércia de rotação são desprezados e que as secções transversais planas permanecem planas e perpendiculares ao eixo longitudinal da viga após o deslocamento, tem-se o modelo

$$
m \frac{\partial^{2} v}{\partial t^{2}}+E I \frac{\partial^{4} v}{\partial x^{4}}+N \frac{\partial^{2} v}{\partial x^{2}}=f(t, x)
$$

\footnotetext{
${ }^{1}$ Projeto UPF-RS. E-mail: giareta@pas.matrix.com.br

${ }^{2}$ Auxílio FAPERGS. E-mail: julio@mat.ufrgs.br
} 


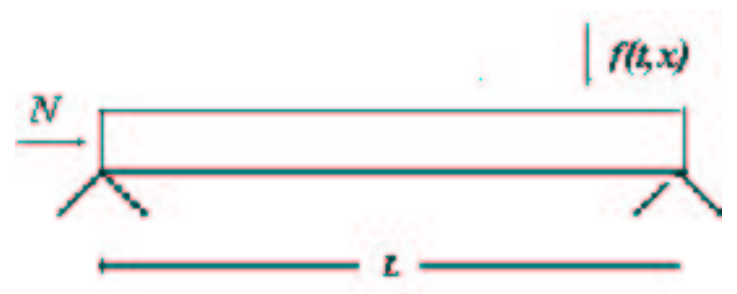

Figura 1: Viga fixa livre sob ação da força axial N

onde $A$ é a área transversal da viga, $E$ o módulo de Young, $I$ o momento de inércia, $N$ a força axial, $\rho$ a densidade linear. Este modelo é de natureza evolutiva, exigindo duas condições iniciais, $v(0, x)=v_{0}(x)$ e $v_{t}(0, x)=\dot{v}_{0}(x)$ que representam o deslocamento e velocidade iniciais da viga, respectivamente, e satisfazendo condições de contorno espaciais, com derivadas até terceira ordem avaliadas em ambas extremidades da viga, $X=0$ e em $x=L$, tais condições podem ser escritas na forma genérica e matricial,

$$
\left[\begin{array}{llllllll}
A_{11} & B_{11} & C_{11} & D_{11} & 0 & 0 & 0 & 0 \\
A_{12} & B_{12} & C_{12} & D_{12} & 0 & 0 & 0 & 0 \\
0 & 0 & 0 & 0 & A_{21} & B_{21} & C_{21} & D_{21} \\
0 & 0 & 0 & 0 & A_{22} & B_{22} & C_{22} & D_{22}
\end{array}\right]\left[\begin{array}{l}
v(t, 0) \\
v_{x}(t, 0) \\
v_{x x}(t, 0) \\
v_{x x x}(t, 0) \\
v(t, L) \\
v_{x}(t, L) \\
v_{x x}(t, L) \\
v_{x x x}(t, L)
\end{array}\right]=\left[\begin{array}{l}
0 \\
0 \\
0 \\
0 \\
0 \\
0 \\
0 \\
0
\end{array}\right] .
$$

\section{Modos Flexurais}

A equação de Euler -Bernoulli pode ser escrita de forma compacta e, análoga a uma equação vibratória conservativa, como

$$
M \ddot{v}+K v=f(t, x),
$$

onde $M=m \mathcal{I}$ com $\mathcal{I}$ o operador identidade e $K$ é o operador diferencial linear espacial de quarta ordem $K=E I \frac{d^{4}}{d x^{4}}+N \frac{d^{2}}{d x^{2}}$, atuando sob funções que satisfazem as condições de contorno (1.2).

Vibrações livres da forma oscilatória $v(t, x)=e^{i \omega t} X(x)$ existem quando $X$ é uma solução não nula da equação modal $\left[K-\omega^{2} M\right] X=0$, isto é,

$$
X^{i v}(x)+g^{2} X^{i i}(x)-a^{4} X(x)=0,
$$


sujeita a condições de contorno. Aqui, os coeficientes são dados por $g^{2}=\frac{N}{E I} \quad \mathrm{e}$ $a^{4}=\frac{m \omega^{2}}{E I}$. Pela propriedade de simetria dos operadores M e K, os modos de vibração satisfazem relações de ortogonalidade.

Escrevendo $X(x)=\Phi c$, onde $\Phi$ denota uma base de soluções [ $\left.\phi_{1} \phi_{2} \phi_{3} \phi_{4}\right]$, temse o sistema $\Delta c=0, \Delta=B \Phi_{o} l$. Introduzindo a solução fundamental $\mathrm{h}(\mathrm{x})$ que satisfaz o problema de valor inicial

$$
h^{i v}(x)+g^{2} h^{i i}(x)-a^{4} h(x)=0
$$

com as condições iniciais $h(0)=h^{\prime}(0)=h^{\prime \prime}(0)=0, h^{\prime \prime \prime}(0)=1$, o conjunto $h, h^{\prime}, h^{\prime \prime}, h^{\prime \prime \prime}$ forma uma base de soluções. Em termos espectrais,

$$
h(x)=\frac{\delta \operatorname{senh} \epsilon x-\epsilon \operatorname{sen} \delta x}{\delta^{2}+\epsilon^{2}},
$$

onde $\delta^{2}-\epsilon^{2}=g^{2}, \delta^{2}=g^{2} / 2+\sqrt{\left(g^{4} / 2-a^{4}\right)}$. Pelos valores iniciais de $\mathrm{h}(\mathrm{x})$, observase que o sistema $\Delta c=0$ pode ser reduzido segundo as condições de contorno do sistema, pois

$$
\Delta=\left[\begin{array}{llll}
A_{14} & A_{13} & A_{12} & A_{11} \\
A_{24} & A_{23} & A_{22} & A_{21} \\
B_{1 k} h^{(k-1)}(L) & B_{1 k} h^{(k)}(L) & B_{1 k} h^{(k+1)}(L) 1 & B_{1 k} h^{(k+2)}(L) \\
B_{2 k} h^{(k-1)}(L) & B_{2 k} h^{(k)}(L) & B_{2 k} h^{(k+1)}(L) 1 & B_{2 k} h^{(k+2)}(L)
\end{array}\right]
$$

onde nas últimas duas linhas é utilizada notação tensorial, abreviação para indicar somatória com variação d o índice $\mathrm{k}=1: 2$. Deve ser observado que as condições iniciais de $h(x)$ independem dos parâmetros da equação, portanto a base dinâmica comporta-se melhor do que a base espectral clássica formada em termos das raízes da correspondente equação caraterística.

\section{A Resposta Livre Através da Função de Green Temporal}

Pelo método espectral de Fourier, a resposta livre da equação do movimento da viga (1.1) pode ser descrita na forma

$$
v(t, x)=\sum_{n=1}^{\infty} v_{n}(t) X_{n}(x)
$$

onde $X_{n}(x)$ são os modos e os $v_{n}(t)$ os coeficientes temporais.

Por substituição na equação homogênea e utilizando a ortogonalidade dos modos, decorre que os coeficientes temporais $v_{n}(t)$ satisfazem à equação

$$
\ddot{v}_{n}(t)+\omega_{n}^{2} v_{n}(t)=0 \text {. }
$$


Multiplicando-se ambos os lados da equação (3.2) no tempo $\tau$ por $h(t-\tau)=$ $\frac{\operatorname{sen} \omega_{n}(t-\tau)}{\omega_{n}}$ e integrando-a por partes em $\tau$ de 0 a $t$, obtém - se

$$
v_{n}(t)=\cos \left(w_{n} t\right) v_{n}(0)+\frac{\operatorname{sen}\left(w_{n} t\right)}{w_{n}} \dot{v}_{n}(0)
$$

Subtituindo-se na série (3.1), decorre que

$$
v(t, x)=\int_{0}^{L}\left[\frac{\partial h}{\partial t}(t, x, s) m v_{0}(s)+h(t, x, s) m \dot{v}_{0}(s)\right] d s,
$$

onde

$$
h(t, x, s)=\sum_{n=1}^{\infty}\left(\frac{X_{n}(s) X_{n}(x)}{\left\|X_{n}\right\|^{2}}\right) \frac{\operatorname{sen} w_{n} t}{w_{n}} \frac{1}{m}
$$

é a solução dinâmica do sistema distribuído, que para $t>0$ corresponde à resposta impulso ou a função de Green de valor inicial. Aqui, $\left\|X_{n}\right\|$ denota a norma integral quadrática do modo $X_{n}$.

A fórmula obtida para $\mathrm{v}(\mathrm{t}, \mathrm{x})$, em termos da função de Green temporal, pode ser escrita na forma compacta

$$
v=\frac{d \mathrm{~h}}{d t}\left[m v_{0}\right]+\mathrm{h}\left[m \dot{v}_{0}\right]
$$

onde h é abreviação do operador dinâmico

$$
\mathrm{h}(t) \phi(x)=\int_{0}^{L} h(t, x, s) \phi(s) d s .
$$

A introdução deste operador permite obter uma estrita analogia com a solução de um sistema conservativo concentrado $M \ddot{v}+K v=f(t)$. Os operadores $\mathrm{h}$ e $\dot{\mathrm{h}}$, possuem como núcleos a função de Green e sua derivada temporal, e podem ser associados com as matrizes $\frac{\operatorname{sen}\left(\sqrt{(} M^{-1} K\right) t}{\left.\sqrt{(} M^{-1} K\right)}$ e $\cos \left(\sqrt{(} M^{-1} K\right) t$ que atuam sobre deslocamentos concentrados espacialmente. O cálculo, igual ao caso concentrado, pode ser realizado através de métodos espectrais e não espectrais. Devido à ortogonalidade dos modos, decorre que para cada modo tem-se a relação

$$
\mathrm{h}(t) X_{n}=\frac{\operatorname{sen}\left(\omega_{n} t\right)}{\omega_{n}} X_{n}
$$

ou seja, são são autofunções do operador $\mathrm{h}(\mathrm{t})$.

A seguir, são apresentadas simulações para a função de Green temporal para uma vigas fixa apoiada, biapoiada e livre-livre em determinados instantes de tempo devido a periodicidade. 


\subsection{Viga Fixa Apoiada}

Tem-se as condições de contorno $v(t, 0)=0, v_{x}(t, 0)=0, v(t, L)=0, v_{x x}(t, L)=0$.

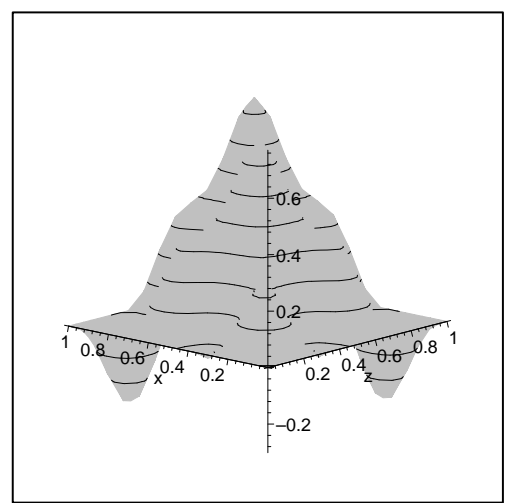

(a)

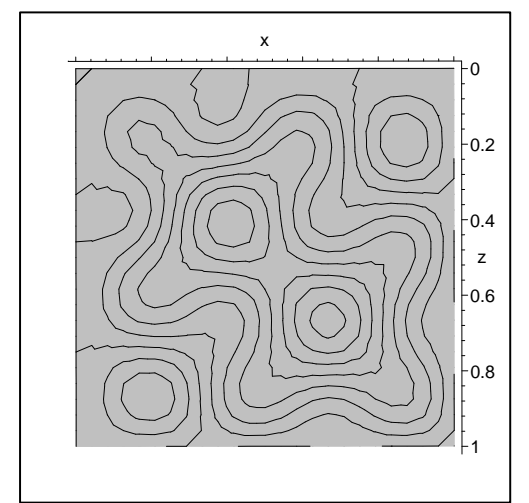

(b)

Figura 2: (a) Gráfico $3 D$ da função de Green em t=3 (b) Linhas de contorno.

\subsection{Viga Biapoiada}

Tem-se as condições de contorno $v(t, 0)=0, v_{x x}(t, 0)=0, v(t, L)=0, v_{x x}(t, L)=0$.

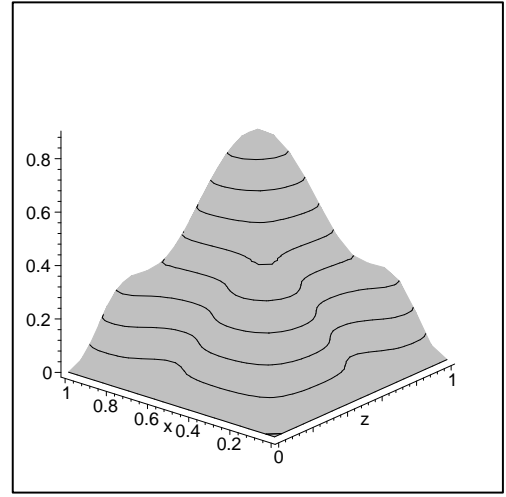

(a)

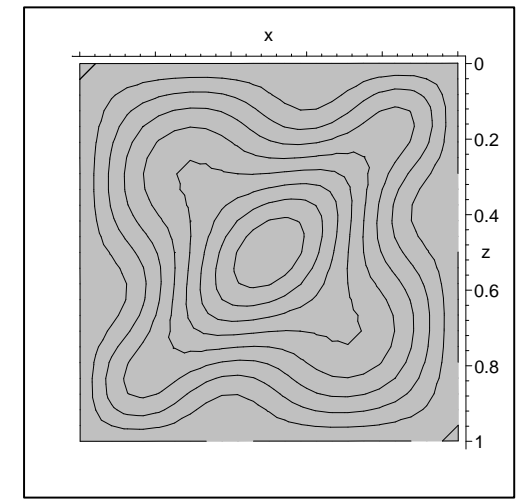

(b)

Figura 3: (a) Gráfico 3D da função de Green em $t=4.4$ (b) Linhas de contorno. 


\subsection{Viga Livre-Livre}

Tem-se as condições de contorno $v_{x x}(t, 0)=0, v_{x x x}(t, 0)=0, v_{x x}(t, L)=0$, $v_{x x x}(t, L)=0$.

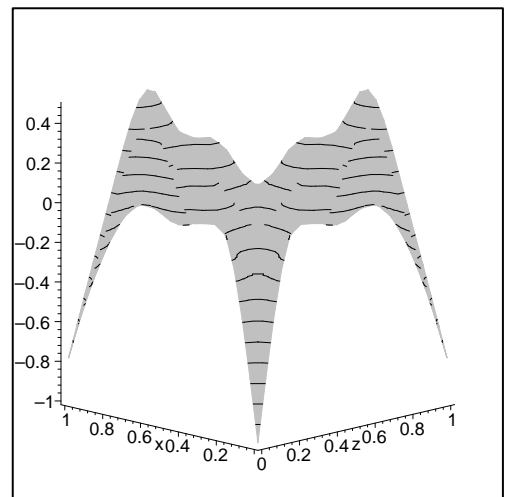

(a)

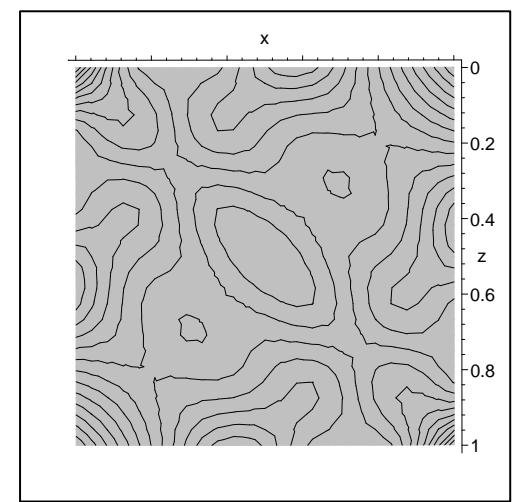

(b)

Figura 4: (a) Gráfico 3D da função de Green em t=3 (b) Linhas de contorno.

\section{Conclusões}

A equação que descreve o modelo de Euler-Bernoulli pode ser considerada de maneira análoga a de um sistema conservativo concentrado em termos da resposta impulso, ou função de Green temporal. Observa-se que a simetria da função de Green em cada instante do tempo é decorrência do fato de o problema modal ser simétrico e que os padrões serem mantidos com o tempo é devido a periodicidade temporal. A formulação utilizada foi direta, evitando a redução para um sistema de primeira ordem, através do espaço de estado, com a qual é obscurecida a simetria do problema.

\section{Referências}

[1] J. Claeyssen, G. Suazo, C. Jung, A direct approach to second-order matrix non-classical vibrating equations, Applied Numerical Mathematics, 30 (1999), 65-78.

[2] J.R. Claeyssen, L.D. Chiwiacowsky, G.S. Suazo, The impulse response in the symbolic computating of modes for beams and plates, Applied Numerical Mathematics, 40 (2002), 119-135. 
[3] D. Findeisen, "System Dynamics and Mechanical Vibrations", Springer-Verlag, 2001.

[4] M.K. Giareta, J.R. Claeyssen, Vibrações forçadas com força axial, em "Aplicon" (J.M. Balthazar et al., eds.), USP, São Carlos, 2001.

[5] J. Ginsberg, "Mechanical and Structural Vibrations", John Wiley, 2001.

[6] A.J. Hull, A closed form solution of a longitudinal bar with a viscous boundary condition, J. Sound Vib., 169, No. 1 (1994), 19-28.

[7] R.A.L. Soder, J.R. Claeyssen, Modos Flexurais sob a Influência de uma Força Axial, em "XXIII CNMAC", Santos, São Paulo, 2000. 
